\title{
A new damping modelling approach and its application in thin wall machining
}

\author{
O. B. Adetoro • P. H. Wen • W. M. Sim
}

Received: 18 June 2009 / Accepted: 6 April 2010/Published online: 29 April 2010

(C) Springer-Verlag London Limited 2010

\begin{abstract}
In this paper, a new approach to modelling the damping parameters and its application in thin wall machining is presented. The approach to predicting the damping parameters proposed in this paper eliminates the need for experiments otherwise used to acquire these parameters. The damping model proposed was compared with available damping models and experimental results. A finite element analysis and Fourier transform approach has been used to obtain frequency response function (FRF) needed for stability lobes prediction. Several predicted stable regions using both experimental and numerical FRF's for various examples gave a good comparison.
\end{abstract}

Keywords FEA · Stability lobes · Transfer function . Frequency response function · High speed milling . Damping ratio $\cdot$ Damping matrix

\section{Introduction}

Chatter, as stated by Taylor as early as 1907 , is one of the most obscure and delicate of all problems facing the machinist [1]. It undermines and reduces productivity and surface quality in manufacturing. There are various sophisticated models to predicting stable regions in highly nonlinear machining processes in the form of a chart.

O. B. Adetoro · P. H. Wen $(\bowtie)$

Department of Engineering, Queen Mary University of London, London E1 4NS, UK

e-mail: p.h.wen@qmul.ac.uk

W. M. Sim

Airbus,

New Filton House, Golf Course Lane,

Filton BS34 7AR, UK
However, new complications are introduced when attempts are made to apply these models to thin wall machining. For rigid workpiece, the dynamic vibrations are from the tool, whose dynamic parameters can be assumed to be constant with very good accuracy as reported by Weck et al. [2]. On the other hand, in thin wall machining, the vibrations mainly come from the workpiece as the tool stiffness is much higher compare to that of the workpiece. Moreover, the workpiece dynamics are constantly changing due to the change in geometry; hence, the difficulty in the accurate modelling of the dynamic parameters. The mass/inertia and stiffness forces can be predicted numerically with very good accuracy for any given geometry, material properties and boundary conditions. Unlike the well-developed mass/ inertia and stiffness terms, the damping forces are, at present, extracted through experiments known as modal testing/analysis. This is because the physics behind the damping forces are not fully understood especially for a wide range of systems. Therefore, in order to consider this change in the workpiece dynamics and, hence, accurately predict stable regions for thin wall machining, a large amount of experimental tests would be required, which is highly impractical.

The prediction of stable conditions in the form of charts started when Tobias [3] and Tlusty [4] simultaneously made the remarkable discovery that the main source of selfexcited regenerative vibration/chatter is not related to the presence of negative process damping as was previously assumed. However, it is related to the structural dynamics of the machine tool-workpiece system and the feedback response between subsequent cuts. Though, a pioneering research, their model is only applicable to orthogonal metal cutting where the directional dynamic milling coefficients are constant and not periodic like in milling. Other studies on the stability of orthogonal metal cutting were reported 
by Merritt [5]. Sridhar et al. [6] later carried out an in-depth study, in which they introduced time-varying directional coefficients in their chatter stability analysis. They used the system's state transition matrix in their stability model, which helps to eliminate the periodic and time delay terms. Slavicek [7] and Vanherck [8] made the assumption that all the cutter teeth have a constant directional orientation in their study of the effect of irregular pitch on the stability. Tlusty [9] made an attempt to apply the orthogonal model to milling process by assuming the teeth of the tool had equal pitch, was simultaneously in cut and that the motion was rectilinear with constant depth of cut. Optiz et al. [10, 11] used an average value of the periodic directional coefficients in the analysis. The Nyquist criterion was used by Minis and Yanushevsky [12, 13] and Lee et al. [14, 15] to obtain the stability limits. Lee et al. [14, 15] used the mean value method to replace the time-varying directional coefficients by a constant. Altintas and Budak [16] later proposed an analytic approach in which the average value in the Fourier series expansion (single frequency solution) of the time-varying coefficient was adopted. This is the main analytical approach generally used in predicting stable cutting conditions in milling [17-20]. Budak and Altintas $[21,22]$ later showed that the results obtained by including the harmonic terms (multi-frequency solution) are very close to the single frequency solution. Campa et al. [23] also considered the mono-frequency solution when considering the milling of thin walls and thin floors. They introduced the averaging of the cutting coefficients and the axial immersion angle along the cutting edge. Adetoro et al. [24, 25] recently proposed a numerical approach to solve this nonlinear coefficients and axial immersion along the cutting edge, although they only considered the singlefrequency solution. Of all these studies, the dynamic parameters of the tool-workpiece system were required and identified experimentally through modal analysis or testing.

Attempts were made by Thevenot [26] to use the varying dynamics in thin wall machining to initiate the variation of the spindle speed along the workpiece in order to improve surface finish. The tendency in this approach, however, is for new marks to be left on the surface due to the change in cutting conditions as seen from their experimental results. Seguy et al. [27] just recently carried out a study to include the varying dynamics along a thin wall and thin floor section, although the results showed certain discrepancies which could have arisen from the assumptions made. It is, however, clear in thin wall machining that it is insufficient to assume that the dynamics of the workpiece are constant, which was the case in previous studies. FEM and Fourier approach to predicting the stable margin presented by Adetoro et al. [28] was recently used to incorporate the changing dynamics in the stability margin prediction by
Adetoro et al. [25]. Their results were validated with good agreement with experimental results. Quintana et al. [29, 30] proposed experimental approaches to predicting the stable margin in milling that does not require the prior extraction of dynamic parameters.

This paper reports a newly discovered relationship between the damping parameters, and the geometry of the structure and proposes an approach that uses identified damping parameters for a known geometry to predict the damping parameters for any geometry with a different thickness. This approach is directly applied to the machining of thin wall sections, thereby eliminating the need for more experimental modal analysis. The proposed approach can be directly used to predict the damping parameters as the workpiece thickness reduces, while mass and stiffness parameters can be modelled numerically with satisfied accuracy. More importantly, it is envisaged that by understanding why this relationship exists for this simple case and other more complex cases could lead to understanding how the damping parameters changes with change in structure's geometry and possible prediction of the damping parameters for any given geometry. Such understanding could perhaps be the definition of the damping parameters for any given geometry in terms of its mass (inertia) terms, stiffness terms and other terms or parameters. The existence of this relationship and the approach is validated using experimentally identified damping parameters, time-domain finite element (FE) simulations of dynamic vibrations and stability lobes predictions for various examples.

\section{Modal analysis}

\subsection{Experimental modal analysis}

A significant contribution at the early development of modal analysis was proportional damping model. It was first proposed by Rayleigh [31] in 1878, where he indicated that if the viscous damping matrix is proportional to mass and stiffness matrices (the damping forces are proportional to the kinetic and potential energies of the system), then it can be expressed as

$[C]=\alpha_{0}[M]+\alpha_{1}[K]$,

where $\alpha_{0}$ and $\alpha_{1}$ are real positive constants. The model is termed 'Rayleigh damping' or 'classical damping'. The significance of this model is that the damped system would have the same mode shapes compared to its undamped counterpart; thus, the system is said to possess 'classical normal modes'. In proportional damping model, the viscous damping matrix in the same manner as the mass and 
stiffness matrices can be generalised using the system's modal matrix or eigenvectors, $\{\Psi\}$. This considerably simplifies the dynamic analysis by allowing a multidegree of freedom (MDoF) system to be represented by a series of uncoupled single degree of freedom (SDoF) systems.

The equation of motion for a MDoF system can be expressed as

$$
[M]\{\ddot{x}(t)\}+[C]\{\dot{x}(t)\}+[K]\{x(t)\}=\{F(t)\},
$$

where $[M]$ is the mass matrix, $[C]$ is the viscous damping matrix, $[K]$ is the stiffness matrix, $\{\ddot{x}(t)\},\{\dot{x}(t)\},\{x(t)\},\{F$ $(t)\}$ are the acceleration, velocity, displacement and excitation force vectors, respectively.

In 1960, Caughey and O'Kelly [32] provided a generalisation of Rayleigh's condition for discrete systems in form of the series

$[C]=[M] \sum_{i=0}^{N-1} \alpha_{i}\left([M]^{-1}[K]\right)^{i}$.

where $N$ is the number of identified modes used in the curve fitting and $a_{i}$ are real positive constants obtained through using experimentally identified damping parameters. The Rayleigh damping model is the first two series of the expansion.

There are different modal testing methods available; however, one of the most common involves the excitation of the structure using an instrumented hammer and measurement of the response with the use of a transducer (either laser based vibration transducers or accelerometers). The impact excitation and the vibration response of the structure are measured and transformed into 'frequency response functions' (FRFs) using a Fourier Analyzer. Subsequently, the structure's dynamic parameters are extracted from the measured FRF. Great contributions on the identification of the dynamic parameters are reported by [33-35], with a good review of the developments reported by [36]. Other notable developments are methods proposed to reduce or eliminate the systematic and noise errors which adversely affect the measured FRFs [37]. One of these systematic errors is the so called mass loading effect which occurs when an accelerometer is used as the transducer. The accelerometer directly changes the dynamics of the system and causes the measured resonant frequencies to deviate from their correct values. Methods of correcting of the mass loading effects on the direct FRFs are reported by [33, 38].

Considering the undamped MDoF system in (Eq. 2) is excited sinusoidally by a set of forces, $\{f(t)\}$, all at the same frequency, $\omega$ but with different amplitudes and phases and assuming a solution $\{x(t)\}$ exists [33] of the form

$\{x(t)\}=\{X\} e^{i \omega t}$, where $\omega$ is the natural frequency of the system and $\{X\}$ is a constant, then the undamped MDoF system is expressed as

$\left([K]-\omega^{2}[M]\right)\{\Psi\}=\{0\}$,

which represents an eigenvalue problem, where $\omega_{n}$ is the eigenvalue (undamped natural frequency squared) and $\{\Psi\}$ is the eigenvector. The characteristic equation of the system is simply

$\left|[K]-\omega_{n}^{2}[M]\right|=0$.

The equation of motion is also represented in Laplace transform domain as follows

$\left([M] s^{2}+[C] s+[K]\right)\{X(s)\}=\{F(s)\}$,

or

$[D(s)]\{X(s)\}=\{F(s)\}$.

where $\{F(s)\}$ is the force in Laplace domain. Hence, the receptance FRF of the system is defined as

$G(s)=\frac{\{X(s)\}}{\{F(s)\}}=[D(s)]^{-1}$,

which rearranges to

$G(s)=\frac{\operatorname{adj}[D(s)]}{|[D(s)]|}$,

where $G(s)$ is the transfer function (TF) matrix of the system and $|[D(s)]|$ is the 'characteristic equation' of the system [39]. The 'characteristic equation' is shared by each element in the transfer function matrix. Therefore, the transfer function matrix can be expressed by its partial fraction expansion as

$\left[G_{i l}(s)\right]=\sum_{p=1}^{N}\left(\frac{r_{i l, p}^{+}}{s-s_{p}^{+}}+\frac{r_{i l, p}^{-}}{s-s_{p}^{-}}\right)$,

where

$s_{p}^{+}, s_{p}^{-}=-\zeta_{p} \omega_{n, p} \pm \sqrt{1-\zeta_{p}^{2}} \omega_{n, p} j$.

where $\zeta_{p}$ is the damping ratio. The complex roots $s_{p}^{+}$and $s_{p}^{-}$and the complex residues $r_{i l, p}^{+}$and $r_{i l, p}^{-}$are a conjugate pair.

Hence,

$\left[G_{i l}(s)\right]=\sum_{p=1}^{N}\left(\frac{\alpha_{i l, p}+\beta_{i l, p} s}{s^{2}+2 \zeta_{p} \omega_{n, p}+\omega_{n, p}^{2}}\right)$,

where, $N$ is the number of modes identified experimentally, $\omega_{n}$ is the natural frequency of the system, $\alpha_{i l, p}$ and $\beta_{i l, p}$ are constants obtained experimentally reflecting the residue of mode $p$ at row $i$ and column $l$. They can be collected into a 
residue matrix $[R]_{p}$ for each mode in order to calculate the mode shapes as explained in [40].

To experimentally measure the direct transfer function $G_{i i}$, the system is excited at point $i$ (using an instrumented hammer), whilst the accelerometer is placed at point $i$. Using a Fourier analyser, the accelerance FRF is extracted for each impact test. This is simply the division of the Fourier transform of the measured time-domain input force $f(t)$ and acceleration $\ddot{x}(t)$.

$A(\omega)=\frac{\ddot{X}(\omega)}{F(\omega)}$.

The use of accelerometer as the transducer alters the frequency response; hence, the error induced in the measured direct FRF is corrected using the expression given by [38]

$A_{\text {new }}(\omega)=\frac{A(\omega)}{1-m_{\text {accel }} A(\omega)}$,

where, $A_{\text {new }}(\omega)$ is the accelerance without the effect of the accelerometer mass, and $m_{\text {accel }}$ is the mass of the accelerometer.

The FRF or TF is simply the imaginary axis, $j \omega$-axis in the $s$-plane (i.e. $s=j \omega$ ). Hence, from Eq. 4, we have that

$x(t)=X e^{i \omega t}$,

$\ddot{x}(t)=(i \omega)^{2} X e^{i \omega t}=-\omega^{2} x(t)$,

Therefore, the receptance FRF, $G(\omega)$ in terms of accelerance FRF is expressed as

$G(\omega)=\frac{X(\omega)}{F(\omega)}=\frac{A_{\text {new }}(\omega)}{-\omega^{2}}$.

This gives the transfer function element $G_{i i}$ in (Eq. 13), where $s=j \omega$. To measure element $G_{i j}$, location $i$ is excited whilst keeping the accelerometer measurement point fixed, thereby giving one row or column of the transfer function matrix. The transfer function matrix obtained is symmetric for a linear system. The experimental measurements are analysed using a modal analysis system, which scans the measured transfer function and fits a curve to the data with a denominator having a $(2 \times N)$-order polynomial, while a transfer function curve is fitted according to Eq. 13. Therefore, the numerical values of natural frequency, damping and residue for each mode are estimated directly [34]. Altintas [40] detailed a method for constructing the full modal matrix of the structure. It should be noted however that Eq. 18 can only be used to remove the mass loading effects for a direct transfer function, $G_{i i}$.

\section{Damping prediction approach}

\subsection{Proposed approach}

This paper proposes a quick, simple and yet accurate approach to predicting the damping ratio in terms of the frequency for a given wall using the known damping ratios of a wall with same height (provided only the wall thickness is changed). For this approach, a new set of parameters, $\bar{\zeta}_{p}$ and $\bar{\omega}_{p}$, are defined as follows

$\bar{\zeta}_{p}=\frac{\zeta_{p}^{a}}{t_{a}}$

for the damping ratio and

$\bar{\omega}_{p}=\frac{\omega_{n, p}^{a}}{t_{a}}$

for the natural frequency, where $t_{a}$ is the reference current wall thickness, $\zeta_{p}^{a}$ is the experimentally identified modal damping ratio and $\omega_{p}^{a}$ is the experimentally identified natural frequency for the reference wall, respectively. These parameters $\left(\bar{\zeta}_{p}\right.$ and $\left.\bar{\omega}_{p}\right)$ are then used to predict the damping ratio, $\zeta_{p}^{b}$, in terms of frequency, $\omega_{p}^{b}$, for any new geometry (provided only the wall thickness is changed) by simply multiplying $\bar{\zeta}_{p}$ and $\bar{\omega}_{p}$ by the new wall thickness $t_{b}$ as follows

$\zeta_{p}^{b}=\bar{\zeta}_{p} \cdot t_{b}$,

$\omega_{p}^{b}=\bar{\omega}_{p} \cdot t_{b}$.

It should be noted that $\zeta_{p}^{b}$ and $\omega_{p}^{b}$ are not necessarily the precise modal damping and natural frequencies of the new wall. To further improve this approach, it is found that $\bar{\zeta}_{p}$ and $\bar{\omega}_{p}$ can be accurately represented by a series expansion expressed as

$\bar{\zeta}=\sum_{i=1}^{N / 2}\left(\alpha_{2 i-1} \cdot \bar{\omega}^{-i}+\alpha_{2 i} \cdot \bar{\omega}^{i}\right)$,

where constants $\alpha_{2 i-1}$ and $\alpha_{2 i}$ are real constants obtained using least squares method. This series expands out in the form

$\bar{\zeta}=\frac{\alpha_{1}}{\omega}+\alpha_{2} \bar{\omega}+\frac{\alpha_{3}}{\omega^{2}}+\alpha_{4} \bar{\omega}^{2}+\frac{\alpha_{5}}{\bar{\omega}^{3}}+\alpha_{6} \bar{\omega}^{3}+\ldots$

Therefore, to obtain the precise damping parameters for each mode for the new wall thickness, the different numerically extracted natural frequencies in (Eq. 6) for the new wall thickness is divided by $t_{b}$ to obtain $\bar{\omega}$ in Eq. 24 and then multiplying the calculated $\bar{\zeta}$ by $t_{b}$, the corresponding damping for that mode is obtained. 


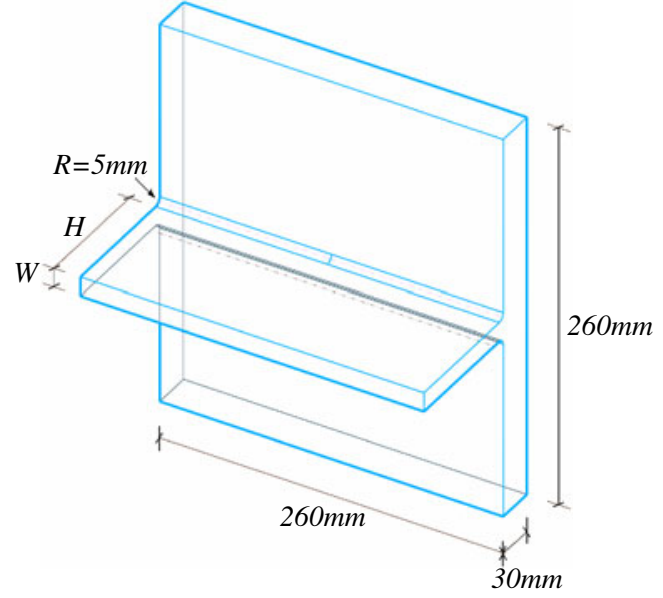

Fig. 1 Workpiece dimensions. corresponding geometry

\subsection{Damping matrix}

The damping ratio, $\zeta_{p}^{b}$ in terms of frequency can be readily used directly by most commercial FE packages; however, the damping matrix $[C]$ in (Eq. 2) is sometimes required. To obtain the damping matrix, the numerically extracted natural frequency of the new structure for each mode is divided by the wall's thickness, $t_{b}$, to obtain $\bar{\omega}$ and used in Eq. 23 to calculate $\bar{\zeta}$, which is then multiplied back by the wall's thickness, $t_{b}$ to obtain the new modal damping ratio for the corresponding mode.

The modal damping $C_{p}$ is simply calculated in a similar fashion to SDoF as

$C_{p}=2 \zeta_{p} \sqrt{k_{p} m_{p}}$,

where $k_{p}$ and $m_{p}$ are the modal stiffness and mass respectively. The damping matrix $[C]$ is finally obtained by pre-multiplying by the modal matrix and then postmultiplying by the transpose of the modal matrix.

Table 1 Case A, $H=30 \mathrm{~mm}, W=4.5 \mathrm{~mm}$

\begin{tabular}{lll}
\hline $\begin{array}{l}\text { Mode number, } \\
P\end{array}$ & $\begin{array}{l}\text { Natural frequency, } \\
\omega_{n, p}(\mathrm{~Hz})\end{array}$ & $\begin{array}{l}\text { Modal damping } \\
\text { ratio, } \zeta_{p}(\%)\end{array}$ \\
\hline 1 & $4.19004 \mathrm{E}+03$ & $2.9639 \mathrm{E}-02$ \\
2 & $4.35164 \mathrm{E}+03$ & $1.98028 \mathrm{E}-02$ \\
3 & $4.87173 \mathrm{E}+03$ & $1.05143 \mathrm{E}-02$ \\
4 & $5.44748 \mathrm{E}+03$ & $8.81198 \mathrm{E}-03$ \\
5 & $6.28131 \mathrm{E}+03$ & $7.11323 \mathrm{E}-03$ \\
6 & $7.33308 \mathrm{E}+03$ & $4.30877 \mathrm{E}-03$ \\
7 & $8.64908 \mathrm{E}+03$ & $4.94934 \mathrm{E}-03$ \\
\hline
\end{tabular}

Table 2 Case B, $H=30 \mathrm{~mm}, W=3.0 \mathrm{~mm}$

\begin{tabular}{lll}
\hline Mode number, & $\begin{array}{l}\text { Natural frequency, } \\
\omega_{n, p}(\mathrm{~Hz})\end{array}$ & $\begin{array}{l}\text { Modal damping ratio, } \\
\zeta_{p}(\%)\end{array}$ \\
\hline 1 & $2.82985 \mathrm{E}+03$ & $2.50934 \mathrm{E}-02$ \\
2 & $3.19785 \mathrm{E}+03$ & $4.23647 \mathrm{E}-03$ \\
3 & $3.40361 \mathrm{E}+03$ & $3.41381 \mathrm{E}-03$ \\
4 & $3.79282 \mathrm{E}+03$ & $5.63088 \mathrm{E}-03$ \\
5 & $4.36370 \mathrm{E}+03$ & $5.64405 \mathrm{E}-03$ \\
\hline
\end{tabular}

\section{The experimental and finite element model}

\subsection{The experimental setup}

A commercial software package called CutPro was used for the experimental tests. The software package was developed by Altintas, and it has within it a 'Modal Analysis' module which was used to extract all the damping ratios from the tap test experimental results. A Dystran instrumented hammer (model 5800B4) with a plastic tip (model $6250 \mathrm{P})$ was used during the experiments, along with a Dystran accelerometer (model 3225F1). Only the direct transfer function $G_{i i}$ was required for the extraction of the damping ratios. Therefore, the accelerometer was placed directly below the impact point, and the impact point was located at a flexible region near one of the two free corners of the wall.

\subsection{The finite element model}

To validate the propose approach, the transient modal dynamic analysis on Abaqus was used, which is presented in detail by Adetoro et al. [28] and summarised here. Being a very well-developed model, the transient modal dynamic analysis gives the linear response of a defined domain as a function of time for a given time-dependent loading. This can be very easily extracted once the modes of the system are available. This is due to the modes being orthogonal,

Table 3 Case C, $H=30 \mathrm{~mm}, W=1.5 \mathrm{~mm}$

\begin{tabular}{lll}
\hline Mode number, $P$ & $\begin{array}{l}\text { Natural frequency, } \\
\omega_{n, p}(\mathrm{~Hz})\end{array}$ & $\begin{array}{l}\text { Modal damping } \\
\text { ratio, } \zeta_{p}(\%)\end{array}$ \\
\hline 1 & $1.31810 \mathrm{E}+03$ & $3.00098 \mathrm{E}-02$ \\
2 & $1.60375 \mathrm{E}+03$ & $2.42216 \mathrm{E}-03$ \\
3 & $1.70662 \mathrm{E}+03$ & $2.49369 \mathrm{E}-03$ \\
4 & $1.90709 \mathrm{E}+03$ & $2.58821 \mathrm{E}-03$ \\
5 & $2.19484 \mathrm{E}+03$ & $1.66906 \mathrm{E}-03$ \\
\hline
\end{tabular}


Table 4 Case I, $H=70 \mathrm{~mm}, W=7.5 \mathrm{~mm}$

\begin{tabular}{lcl}
\hline Mode number, $P$ & $\begin{array}{l}\text { Natural frequency, } \\
\omega_{n, p}(\mathrm{~Hz})\end{array}$ & $\begin{array}{l}\text { Modal damping } \\
\text { ratio, } \zeta_{p}(\%)\end{array}$ \\
\hline 1 & $1,272.5000$ & $1.6636 \mathrm{E}-02$ \\
2 & $1,465.1000$ & $1.4675 \mathrm{E}-02$ \\
3 & $2,997.7000$ & $8.3671 \mathrm{E}-03$ \\
4 & $2,013.9536$ & $1.0525 \mathrm{E}-02$ \\
5 & $4,378.7000$ & $5.8279 \mathrm{E}-03$ \\
\hline
\end{tabular}

thereby rendering the system as a mere combination of single degree of freedom systems. The modes are extracted in a frequency extraction analysis, which utilises the Lanczos algorithm. The algorithm is detailed by Grimes et al. [41] and in the Abaqus user manual [42]. Therefore, when the model is projected onto the eigenmodes used for the system's dynamic representation (i.e. uncoupling the system's stiffness, mass and damping matrices using the orthogonality property explained earlier), its equation of motion is uncoupled, and an expression at time $t$ [42] is obtained

$\ddot{q}_{p}+2 \zeta_{p} \omega_{n, p} q_{p}+\omega_{n, p}^{2} q_{p}=f_{t-\Delta t}+\frac{\Delta f}{\Delta t} \Delta t$,

where $p$ is the mode number, $q_{p}$ is the amplitude of the response of mode $p$ (in the "generalised coordinate"), $\omega_{n, p}$ is the undamped natural frequency of mode $p, \Delta f$ is the change in $f$ over the time increment, $\Delta t$ assuming the excitation varies linearly within each increment and $\zeta_{p}$ is the damping ratio for mode $p$.

The solutions is obtained [42] in the form

$$
\left\{\begin{array}{l}
q_{t+\Delta t} \\
\dot{q}_{t+\Delta t}
\end{array}\right\}=\left[\begin{array}{ll}
d_{11} & d_{12} \\
d_{21} & d_{22}
\end{array}\right]\left\{\begin{array}{l}
q_{t} \\
\dot{q}_{t}
\end{array}\right\}+\left[\begin{array}{ll}
e_{11} & e_{12} \\
e_{21} & e_{22}
\end{array}\right]\left\{\begin{array}{c}
f_{t} \\
f_{t+\Delta t}
\end{array}\right\}
$$

where $i, l=1,2, d_{i l}$ and $e_{i l}$ are constants, which are dependent on the three different cases of non-rigid body motion. These

Table 5 Case II, $H=70 \mathrm{~mm}, W=3.5 \mathrm{~mm}$

\begin{tabular}{lrl}
\hline Mode number, $P$ & $\begin{array}{l}\text { Natural frequency, } \\
\omega_{n, p}(\mathrm{~Hz})\end{array}$ & $\begin{array}{l}\text { Modal damping } \\
\text { ratio, } \zeta_{p}(\%)\end{array}$ \\
\hline 1 & 575.7263 & $9.2024 \mathrm{E}-03$ \\
2 & 654.2049 & $6.0351 \mathrm{E}-03$ \\
3 & 891.3218 & $5.1987 \mathrm{E}-03$ \\
4 & $1,278.8779$ & $6.0564 \mathrm{E}-03$ \\
5 & $1,849.9236$ & $3.2948 \mathrm{E}-03$ \\
\hline
\end{tabular}

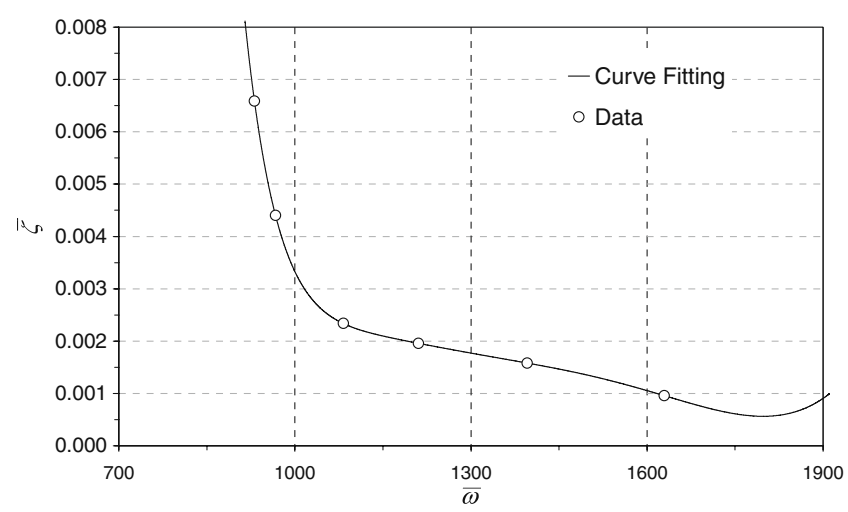

Fig. 2 Comparison between curve fitting using proposed series and $\bar{\zeta}_{p}$

cases are based on the oscillation modes - underdamped, critical damping and overdamped. These constants are detailed in Abaqus user manual [32] and in the paper by Adetoro et al. [28], for the underdamped case.

Since the time integrations is done in generalised coordinates, the response of the physical variables are obtained through summation

$u=\sum_{p}^{N} X_{p} q_{p}$

where $X_{p}$ is the eigenvector corresponding to the mode $p$ and $u$ is the actual nodal displacement. From this, the velocity and, hence, the nodal acceleration can be derived.

The workpiece material used in the FEM model is "Aluminium Alloy 7010T7651". The material properties required for generating the stiffness and mass matrices are: density, $\rho\left(\mathrm{Kg} \mathrm{m}^{-3}\right) 2.823 \times 10^{3}$, Young's Modulus, $E(\mathrm{GPa})$ 69.809 and Poisson Ratio, $v$ 0.337. The workpiece was modelled using second-order reduced integration isoparametric elements. Three different types of workpiece were used in the finite element analysis (FEA). The dimensions are shown in Fig. 1, and the corresponding wall heights, $H$ and thicknesses, $W$ are given in Tables 1, 2, 3, 4 and 5 along with their experimentally identified damping ratios. The damping parameters for Case A (Table 1) and Case I (Table 4) were used to predict the damping parameters, $\zeta_{p}^{b}$ for the remaining structures using the proposed approach in Eq. 25 for Cases B, C and II, respectively. The damping parameters predicted, $\zeta_{p}^{b}$ and the force data, $\mathrm{f}(t)$ measured by the instrumented hammer (in time domain) during impact tests were used in each corresponding FE analysis. The workpiece was bolted at the back surface to a milling machine table during the impact tests; hence, in the FEM simulations, it was assumed to be perfectly clamped and that the resonant frequency of the machine is much higher than the excited frequencies during impact tests. 
Fig. 3 Comparison between predicted damping ratios (using the proposed approach), curve fittings (using Caughey's series and Rayleigh's damping model) and experimental damping ratios: (a) Case A; (c) Case B; (c) Case $\mathrm{C}$

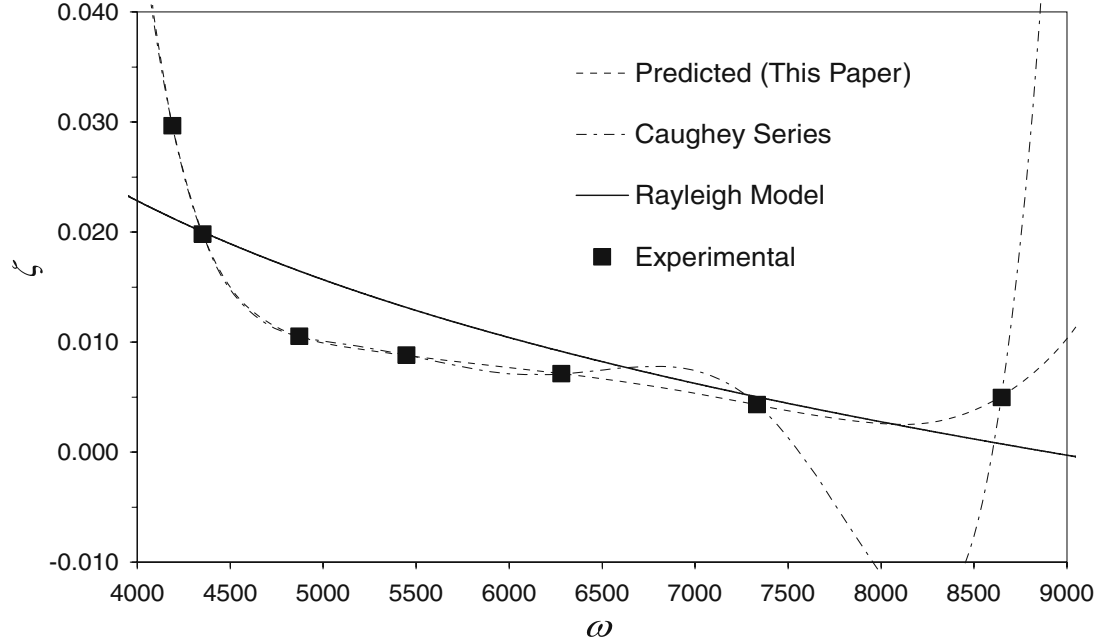

(a) - Case A

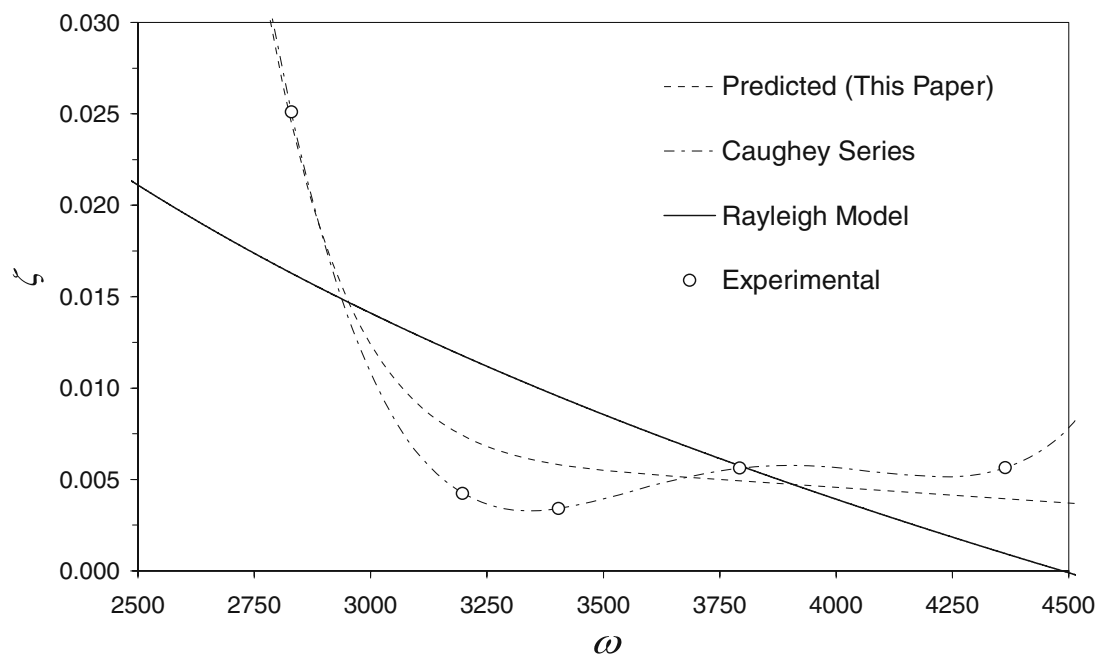

(b) - Case B

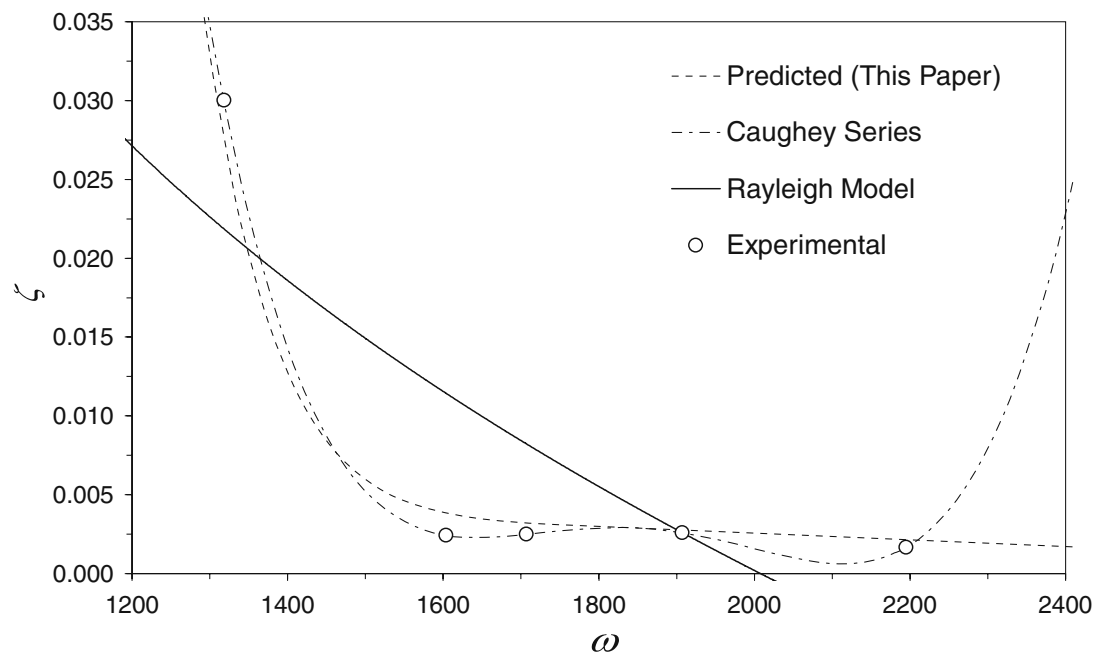

(c) - Case C 
Table 6 Case A

\begin{tabular}{|c|c|c|c|c|c|c|c|}
\hline & $\alpha_{0}$ & $\alpha_{1}$ & $\alpha_{2}$ & $\alpha_{3}$ & $\alpha_{4}$ & $\alpha_{5}$ & $\alpha_{6}$ \\
\hline Rayleigh & 114.4497 & $-1.4440 \mathrm{E}-06$ & & & & & \\
\hline Caughey's Series & $3.5333 \mathrm{E}+04$ & $-2.0794 \mathrm{E}-02$ & $1.1485 \mathrm{E}-05$ & $-2.8317 \mathrm{E}-09$ & $3.6923 \mathrm{E}-13$ & $-2.4854 \mathrm{E}-17$ & $6.8192 \mathrm{E}-22$ \\
\hline
\end{tabular}

\section{Validation}

\subsection{Damping ratio}

\subsubsection{Case 1}

Using the experimental modal analysis explained in Section 2, the different damping ratios were extracted for
Cases A, B and $\mathrm{C}$ as given in Tables 1,2 and 3. The proposed damping prediction approach uses the damping ratio obtained from one geometry (Case A) to predict the damping for any given geometry with only the thickness changed (Cases B and C). Using the least squares method, the damping ratios identified for Case A (see Table 1) were used to extract the coefficients in Eq. 23 as follows:

$$
\begin{array}{ll}
\alpha_{1}=-1.12722 \times 10^{4}, & \alpha_{4}=-1.80546 \times 10^{-6}, \\
\alpha_{2}=3.69315 \times 10^{-3}, & \alpha_{5}=1.21876 \times 10^{10} \\
\alpha_{3}=1.81938 \times 10^{7}, & \alpha_{6}=2.81899 \times 10^{-10}
\end{array}
$$

The curve fitting is compared with the calculated $\bar{\zeta}_{p}$ and $\bar{\omega}_{p}$ in Fig. 2. From this curve fitting, the damping for a new wall thickness can be predicted as shown in Fig. 3a-c for Cases B and C, respectively, using Eqs. 21 and 22.

The experimental data for each geometry (Tables 1,2 and 3) was used to extract the coefficients $a_{j}$ in Rayleigh's damping model and Caughey's series in Eqs. 1 and 3. Rayleigh's damping model can be expressed in generalised coordinate as:

$\zeta_{p}=\frac{1}{2}\left(\frac{\alpha_{0}}{\omega_{n, p}}+\alpha_{1} \omega_{n, p}\right)$,

while Caughey's series can be expressed in generalised coordinates as

$\zeta_{p}=\frac{1}{2}\left(\frac{\alpha_{0}}{\omega_{n, p}}+\alpha_{1} \omega_{n, p}+\alpha_{2} \omega_{n, p}^{3}+\alpha_{3} \omega_{n, p}^{5}+\ldots\right)$.

Hence, using least square method, the experimental damping ratios for each geometry in Tables 1, 2 and 3 were used to extract the coefficients for each corresponding geometry.

Table 7 Case B
The damping ratio curve fittings using both Rayleigh and Caughey's series are compared with the damping ratios from the proposed series in Fig. 3a-c. The comparison between the proposed damping prediction approach, Caughey's series curve fitting and experimental data, is seen to be satisfactory. Though it should be noted that to obtain Caughey's series curve, the experimental data is required for each geometry in order to obtain the coefficients. The coefficients identified for Rayleigh damping model and Caughey's series are given for each geometry in Tables 6, 7 and 8 .

The proposed approach apart from predicting the damping ratio can be used to give a rough estimate of the frequency range of the most significant modes, when performing a FEA or an impact test by using the calculated $\omega_{p}^{b}$. Taking $\bar{\omega}_{1}=882 \mathrm{~Hz}$ for example, it will become $\omega_{1}^{b}=6615 \mathrm{~Hz}$ for a $7.5-\mathrm{mm}$ thick wall using Eq. 22. Using FEM, the first natural frequency is calculated to be $6588.4 \mathrm{~Hz}$; hence, $\omega_{1}^{b}$ can be used as a rough estimate of the minimum frequency of interest and knowledge of the minimum frequency can be used to reduce the computational time in the Lanczos Algorithm. Similarly, by using the maximum frequency of the

\section{Table 7 Case B}

\begin{tabular}{lrrrrr}
\hline & \multicolumn{1}{c}{$\alpha_{0}$} & \multicolumn{1}{c}{$\alpha_{1}$} & $\alpha_{2}$ & $\alpha_{3}$ & $\alpha_{4}$ \\
\hline Rayleigh & 76.5198 & $-3.8016 \mathrm{E}-06$ & & & \\
Caughey's Series & $5,475.3499$ & $-3.8644 \mathrm{E}-03$ & $2.0391 \mathrm{E}-06$ & $-4.0144 \mathrm{E}-10$ & $2.7971 \mathrm{E}-14$ \\
\hline
\end{tabular}


Table 8 Case $\mathrm{C}$

\begin{tabular}{llllll}
\hline & $\alpha_{0}$ & $\alpha_{1}$ & $\alpha_{2}$ & \\
\hline Rayleigh & 925.4978 & $3.6906 \mathrm{E}-05$ & & & \\
Caughey's Series & $1,858.9610$ & $-5.5559 \mathrm{E}-03$ & $+6.0342 \mathrm{E}-06$ & $-2.4443 \mathrm{E}-09$ & $+3.5011 \mathrm{E}-13$ \\
\hline
\end{tabular}

dominant modes in Eq. 22, $\omega_{p}^{b}$ can be taken as a rough estimate of the maximum frequency of the dominant modes for the new structure.

\subsubsection{Case 2}

A wall section with a different height of $70 \mathrm{~mm}$ (Case 2) was further used to validate the proposed approach. The different damping ratios extracted for Cases I and II are given in Tables 4 and 5. Using the least squares method, the damping ratios identified for Case I were used to extract the coefficients in Eq. 23 as follows: $\alpha_{1}=3.68005 \times 10^{-1}$, $\alpha_{2}=3.04763 \times 10^{-7}$, while the curve fitting is compared with the calculated $\bar{\zeta}_{p}$ and $\bar{\omega}_{p}$ in Fig. 4 . From this curve fitting, the damping for a new wall thickness can be predicted as shown in Fig. 5b for Case II using Eqs. 21 and 22.

Rayleigh and Caughey's series coefficients extracted using damping parameters in Tables 4 and 5 are given in Tables 9 and 10. The damping ratio curve fittings using both Rayleigh and Caughey's series are also compared with the damping ratio curve fitting from the proposed series in Fig. 5a, b. The comparison between the proposed damping prediction approach, Rayleigh model and experimental data is satisfactory. The comparison between the proposed series damping model (Eq. 24) and Rayleigh's model in Fig. 5b are exactly the same, because the first two terms in the series in Eq. 24 is equivalent to Rayleigh's model, when $\bar{\zeta}_{p}$ and $\bar{\omega}_{p}$ are converted back into the $\bar{\zeta}_{p}^{b}$ and $\bar{\omega}_{p}^{b}$.

\subsection{Time domain}

\subsubsection{Case 1}

Cases $\mathrm{B}$ and $\mathrm{C}$ were simulated in a commercial FEM package (Abaqus) using the simulation approach explained by Adetoro et al. [28]. Two different simulations were performed for each structure. In the first simulation (for each case), the damping ratios extracted experimentally were used directly. This simulation shows how accurate the actual FEM predictions are compared to experimental results. While in the second simulation (for each case), the predicted damping ratios using the new approach proposed in this paper were used. It should be noted that this second simulation requires no experimental input/data as all the material properties are known and the damping ratios are predicted using the proposed approach. The input force in time-domain $f(t)$ used in both simulations was the impact force measured using the instrumented hammer for each corresponding structure. FEM results for Cases A and I are not included in these results as they would only be comparing the FEM predictions using experimentally extracted damping ratios and the experimental results. Although this will show the accuracy of the FEM approach, the accuracy is already depicted as mentioned earlier in all of the first simulations and also the results presented by Adetoro et al. [28].

The damping ratio $\zeta_{p}$, for the first simulation was the experimental damping ratio for each natural frequency
Fig. 4 Comparison between curve fitting using proposed series and $\bar{\zeta}_{p}$

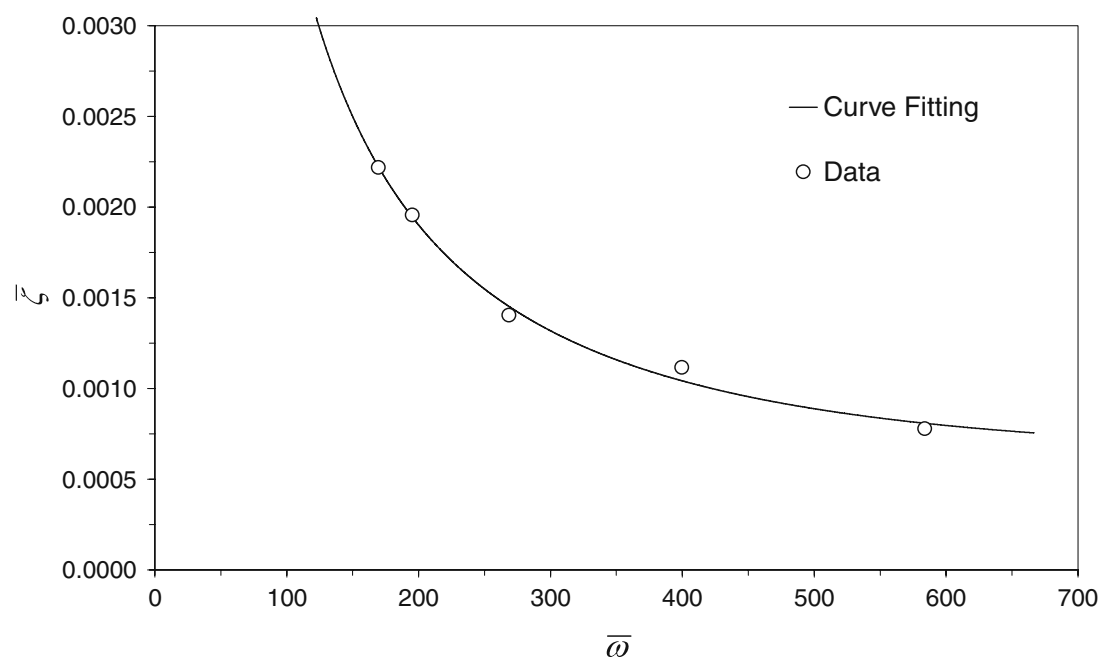


Fig. 5 Comparison between predicted damping ratios (using the proposed approach), curve fittings (using Caughey's series and Rayleigh's damping model) and experimental damping ratios: (a) Case I; (b) Case II

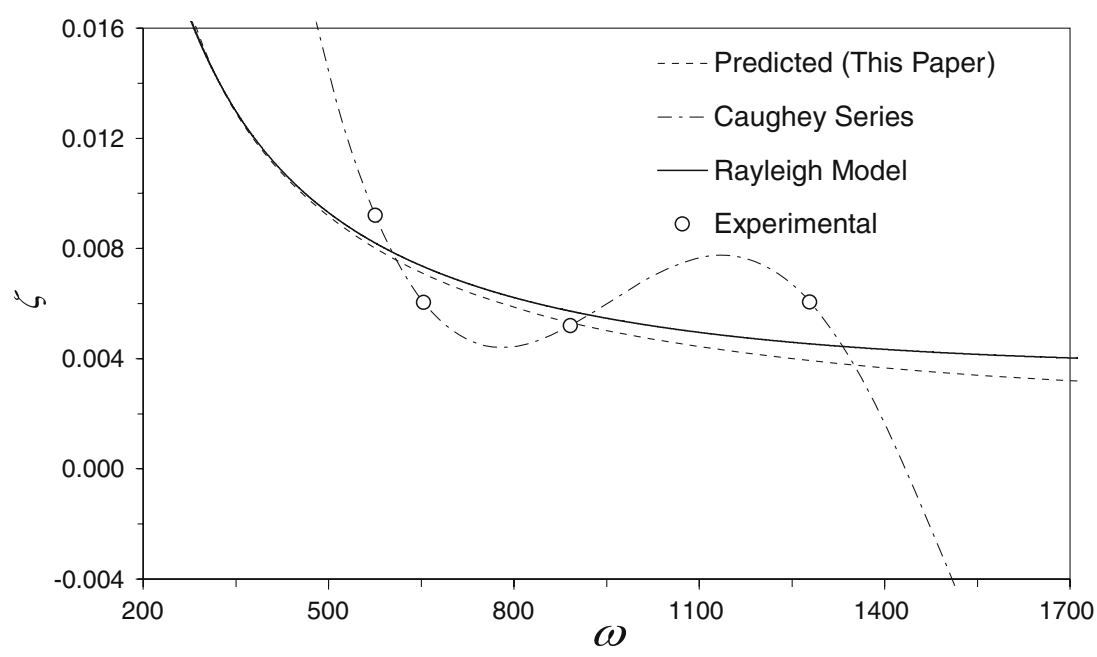

(a) - Case I

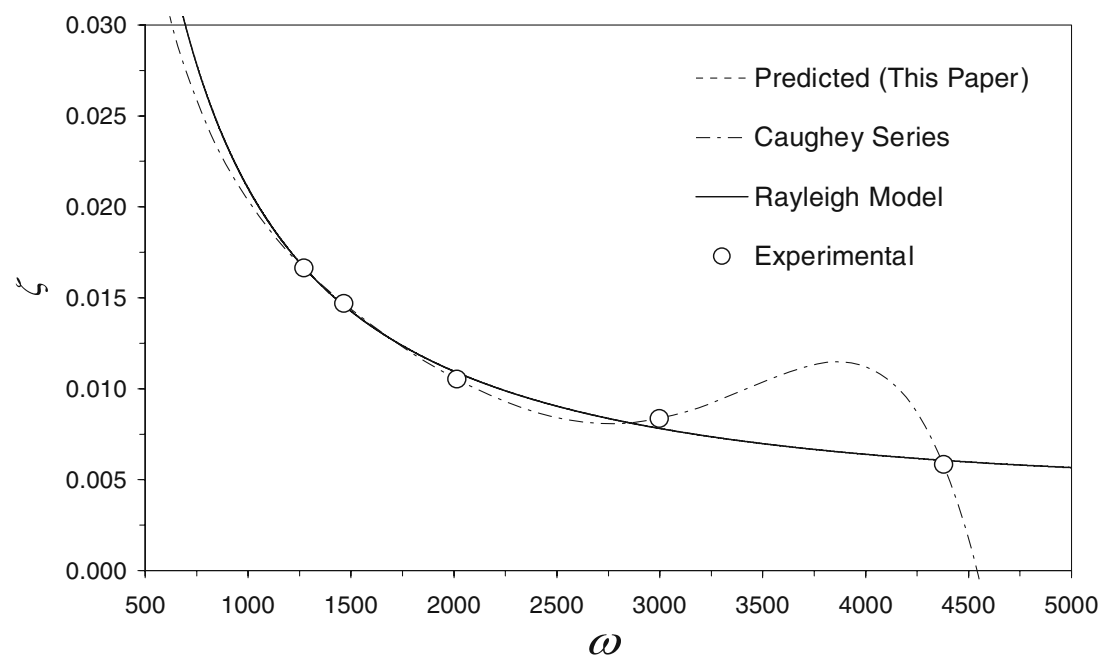

(b) - Case II
(Tables 2 and 3) and in the second simulation corresponds to the predicted damping ratio, $\zeta_{p}^{b}$ (Fig. $3 \mathrm{~b}$ and c), where $\omega_{p}^{b}$ is the numerically identified natural frequency for the structure. The damping ratios are defined in Abaqus in terms of their corresponding frequencies. The acceleration at the same accelerometer location (as it was during the experimental impact testing) was monitored in the $\mathrm{FE}$ analyses and is compared with acceleration measured during the experiments.

Comparison between the FEM modal analysis and the experimental results is satisfactory as shown in Fig. 5a, b.
The comparison between the FEM results when using experimental damping ratios and when using predicted damping ratios show a perfect match (Fig. 6a, b).

\subsubsection{Case 2}

Likewise for Case II, the experimentally identified damping ratios and damping ratio predicted (using the proposed approach) were used in FEM simulations and comparisons to experimental results are shown in Fig. 7 to further show the accuracy of the predicted damping ratios.

Table 9 Case I

\begin{tabular}{llllll}
\hline & $\alpha_{0}$ & $\alpha_{1}$ & $\alpha_{2}$ & $\alpha_{3}$ & $\alpha_{4}$ \\
\hline Rayleigh & 41.4005 & $6.0953 \mathrm{E}-07$ & & & \\
Caughey's Series & 35.2409 & $7.5600 \mathrm{E}-06$ & $2.3704 \mathrm{E}-12$ & $2.6609 \mathrm{E}-19$ & $8.3855 \mathrm{E}-27$ \\
\hline
\end{tabular}


Table 10 Case II

\begin{tabular}{llllll}
\hline & $\alpha_{0}$ & $\alpha_{1}$ & $\alpha_{2}$ & $\alpha_{3}$ & $\alpha_{4}$ \\
\hline Rayleigh & 8.8871 & $1.6646 \mathrm{E}-06$ & & & \\
Caughey's Series & 38.1047 & $1.3808 \mathrm{E}-04$ & $1.9756 \mathrm{E}-10$ & $9.9746 \mathrm{E}-17$ & $1.5534 \mathrm{E}-23$ \\
\hline
\end{tabular}

\subsection{Workpiece transfer function}

The predicted FRF (using the approach detailed by Adetoro et al. [28]) and experimental FRF are compared in Figs. 8, 9 and 10 , respectively. The agreement between the experimental results and the predictions is satisfactory.

\subsection{Chatter stability lobes}

Using both the predicted and experimental FRFs, the stability lobes was generated using CutPro for the different types of workpiece using the parameters listed in Table 11. CutPro is an analytical and time-domain machining process simulation commercial package developed by Altintas. It has a built-in modal analysis module and also a stability lobes module. The stability lobes module can take the transfer function in all three orthogonal directions for the workpiece and transfer function in $\mathrm{x}$ and $\mathrm{y}$ directions for the tool. The predicted and experimental results are compared in Figs. 11b, c and 12 for three different workpieces. The comparisons show a satisfactory agreement. The slight discrepancy in the predicted natural frequency (frequency at which FRF real is zero and imaginary is maximum) can be seen as a slight shift in the spindle speed calculated in the stability lobes. The natural frequency predicted affects the stable tooth passing
Fig. 6 Comparison between FEM predicted acceleration for both simulations and experimentally measured acceleration

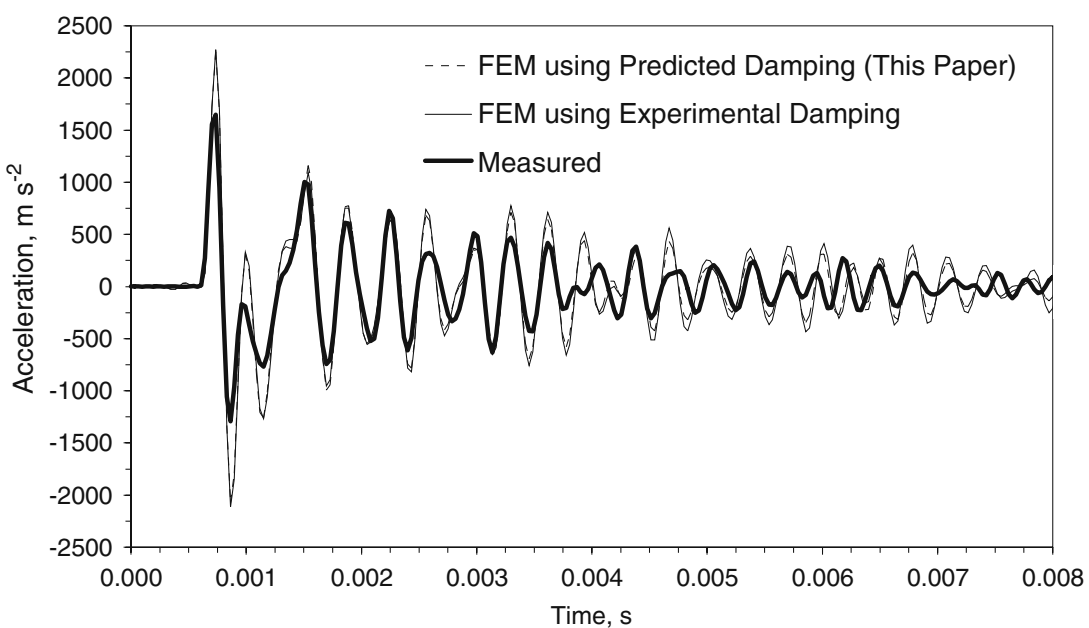

(a) - Case B

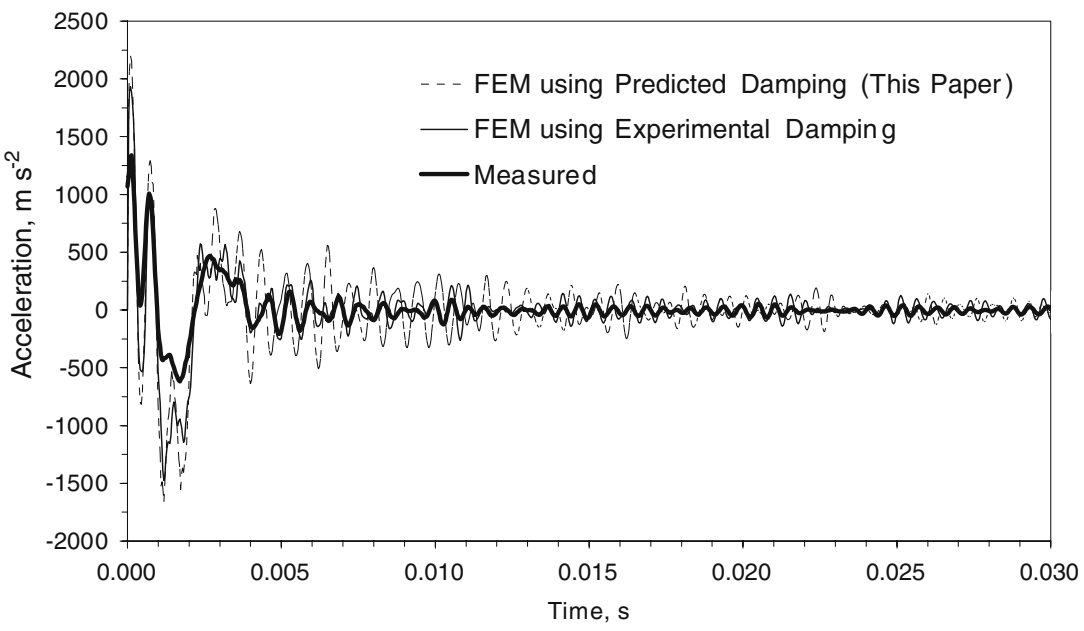

(b) - Case C 


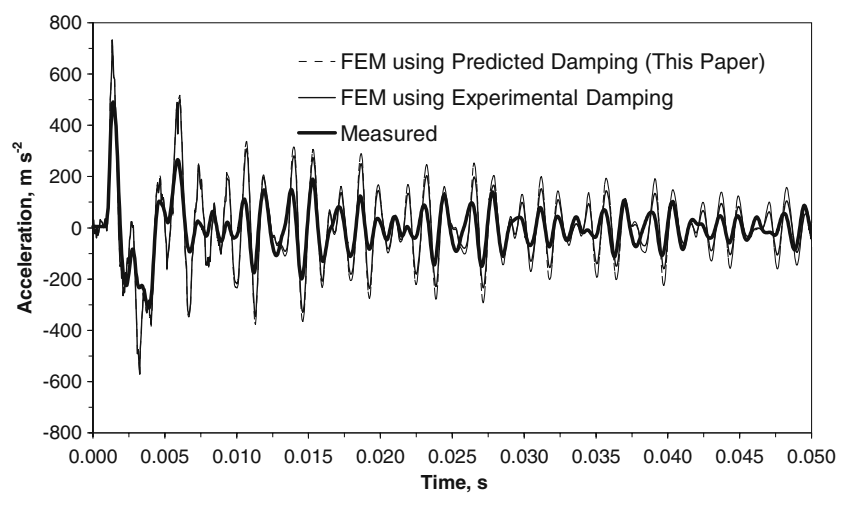

Fig. 7 Comparison between FEM predicted acceleration for both simulations and experimentally measured acceleration

frequency calculated in the stability lobes, hence the slight differences seen in the spindle speeds. The predicted stable axial depths of cuts are slightly higher than the experimental stable $\mathrm{ADOC}$ in case $\mathrm{B}$, and this is due to the FEM model being too stiff. This can be caused by the boundary condition assumption stated in Section 3, where the back surface was assumed to be perfectly clamped. In the FEM stiffness matrix formulation, the elements are, therefore, set to $1 \mathrm{E}+36$, and the degrees of freedom at this surface are not

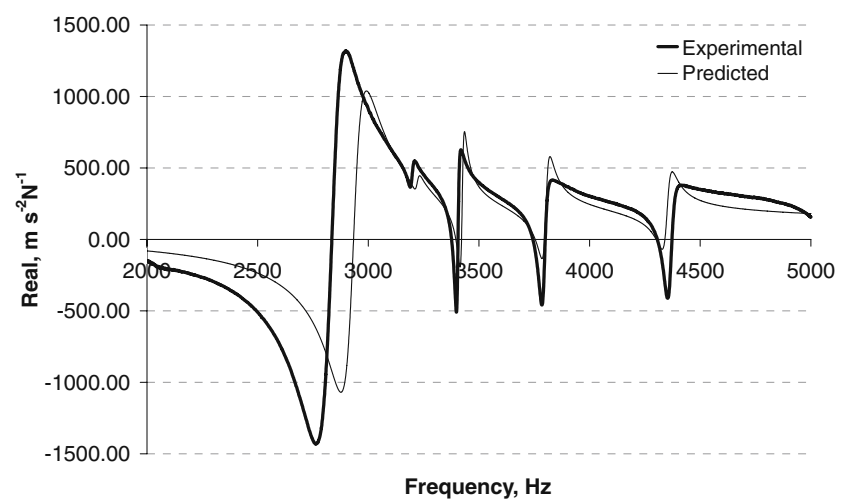

(a) Real

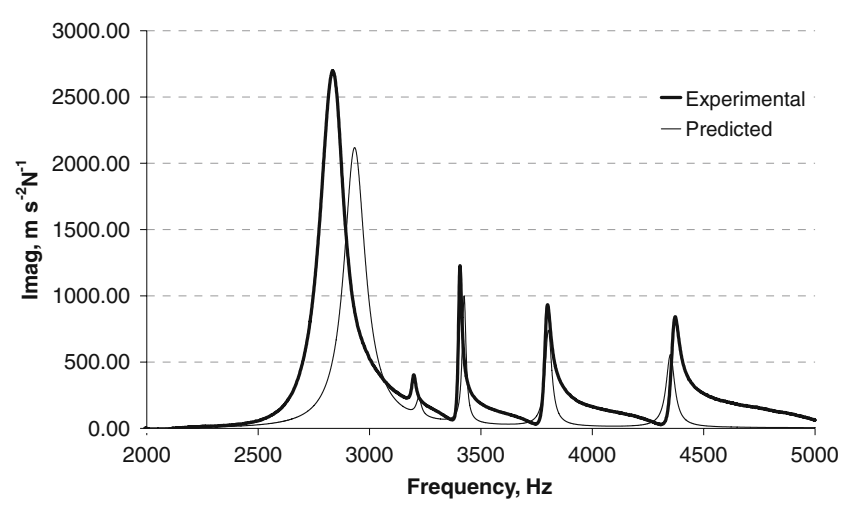

(b) Imag

Fig. 8 Predicted and measured FRFs for Case B, $G_{w_{y y}}$

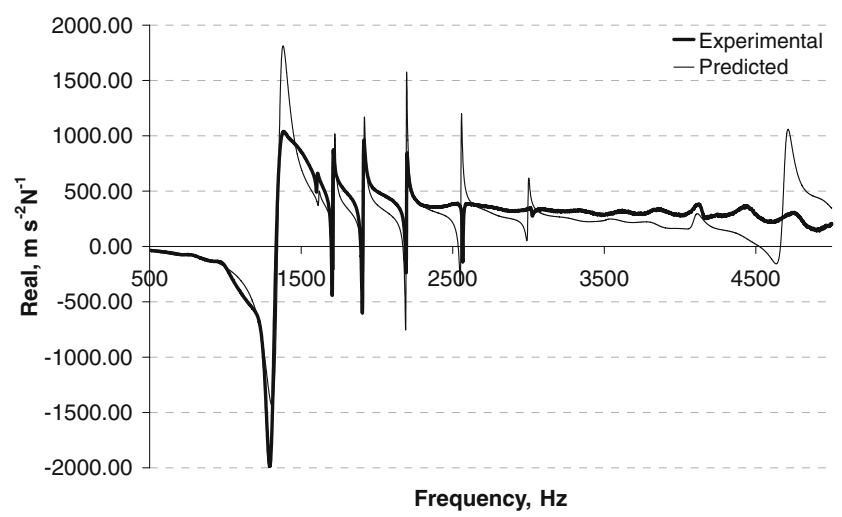

(a) Real

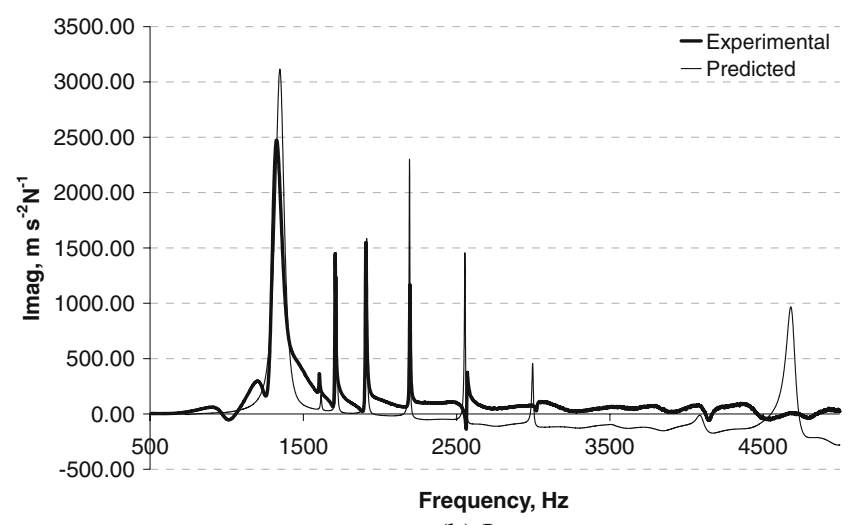

(b) Imag

Fig. 9 Predicted and measured FRFs for Case C, $G_{w_{y y}}$

included in the simulation. A more accurate approach would require knowledge of the friction at the boundary between the machine and the workpiece. There is a difference in the axial depth of cut at a spindle speed of 27,500 rpm in both Cases B and C. The cause of this is unclear, although it may again be due to the predicted damping ratio for a particular mode being too low compared to the experimental modal damping ratio. This

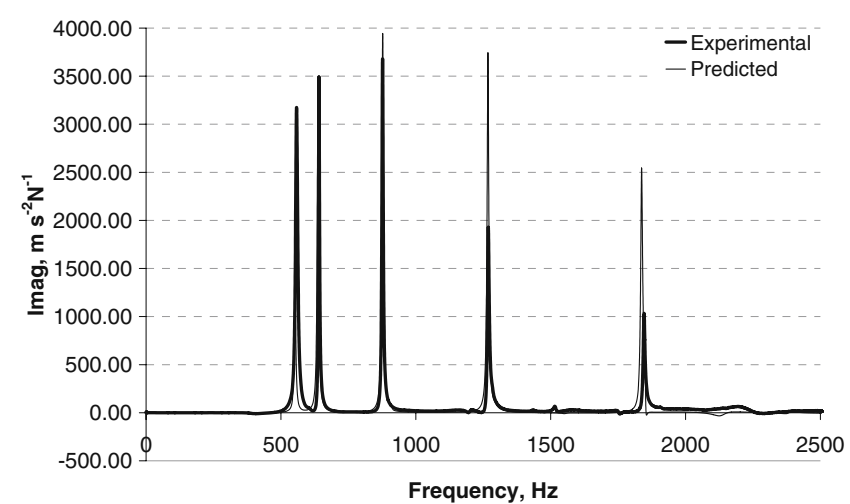

Fig. 10 Predicted and measured FRFs for Case II, $G_{w_{y y}}$ 
Table 11 Force coefficients and radical depths of cut

\begin{tabular}{lccc}
\hline & Case B & Case C & \multicolumn{1}{c}{ Case II } \\
\hline RCFC, $K_{r}$ & 0.3030 & -0.7040 & -0.7040 \\
TCFC, $K_{t}$ (MPa) & 801.0970 & 981.6966 & 981.6966 \\
Radial depth of Cut, $(\mathrm{mm})$ & 1.000 & 0.500 & 0.500 \\
\hline
\end{tabular}

$R C F C$ radial cutting force coefficient, $T C F C$ tangential cutting force coefficient

could also explain why there is a general overestimation in the predicted acceleration in Fig. 6.

The possibilities of predicting the damping ratio for any given structure with a change in only its height was also investigated. The results, although inconclusive, show a possible new set of parameters of the kind.

$\bar{\zeta}_{h}=\zeta_{p}^{a} \cdot h_{a}$

$\bar{\omega}_{h}=\omega_{n, p}^{a} \cdot h_{a}$

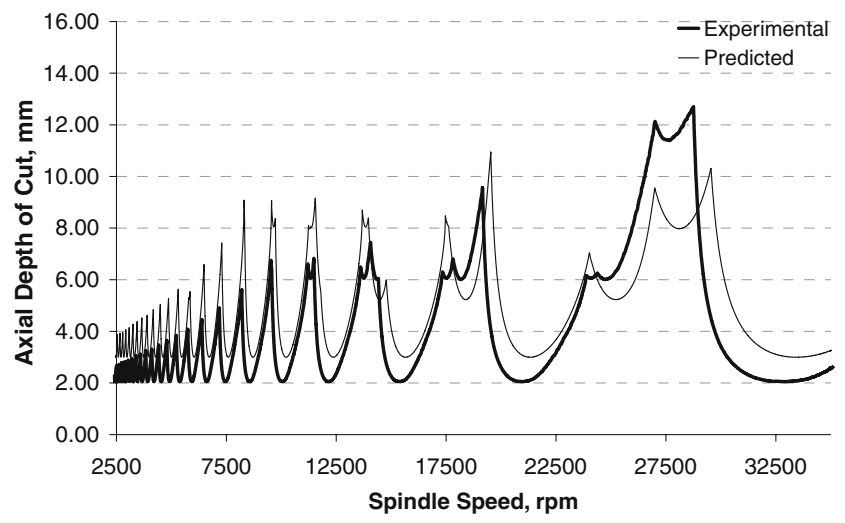

(a) Case B

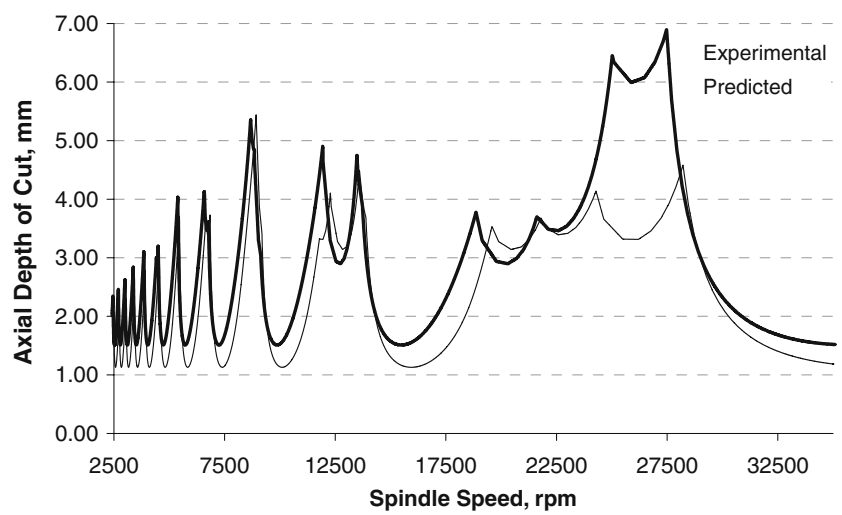

(b) Case C

Fig. 11 Stability lobes comparison

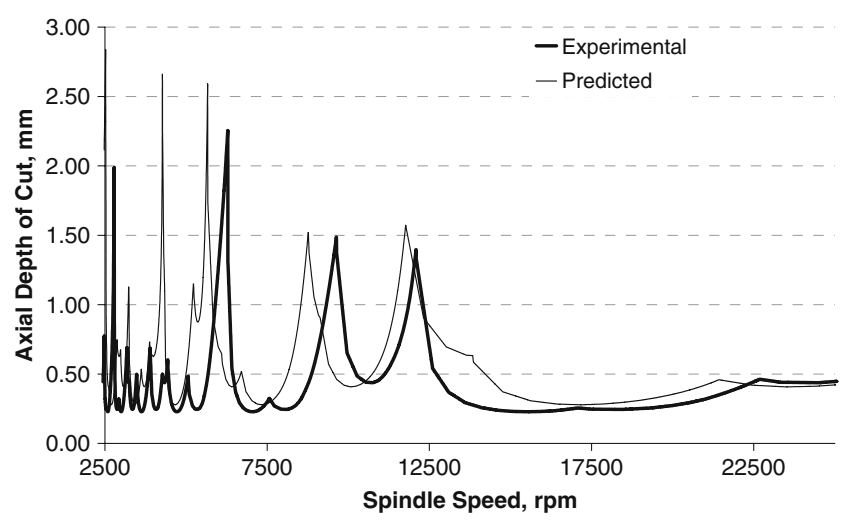

Fig. 12 Stability lobes comparison for Case II

Further experimental tests would, however, be required to further investigate any possible relationship.

\section{Conclusion}

The damping term compared to the stiffness and the mass terms in the equation of motion is far from being understood, while its influence cannot be neglected in nearly all dynamic analyses. This paper proposes a novel approach to using the known damping parameters for a structure to predict the damping parameters for any other geometry provided only the thickness is changed. The approach uses a newly defined set of parameters and also proposes the use of a series to fit a curve to the defined parameters. The model is validated by comparing the predicted damping ratios with experimentally identified damping ratios, which shows a satisfied agreement. Its accuracy is further explored by using the predicted damping ratios in FE time-domain vibration simulations and comparing predicted acceleration with experimentally measured acceleration. The comparison shows a satisfactory match between the FEM predictions and the experimental accelerations. In addition, the proposed approach was applied to thin wall machining, where the dynamic parameters of the workpiece are constantly changing as the workpiece gets thinner. The approach eliminates the need for experiments every time a layer of material is machined. Though a starting point, this approach can be further investigated to further understand the damping ratio variations with the whole structures geometry. This could lead to the prediction of the damping terms for more complicated geometries, with the ultimate aim to reduce the need for or the reliance on experiments. 
Acknowledgements The authors acknowledge the support given by EPSRC for funding this project, the continuous support given by Airbus and also the contributions made by Mr. Alister Reynish from GKN Aerospace.

\section{References}

1. Taylor FW (1907) On the art of cutting metals. Trans Am Soc Mech Eng 28:31-350

2. Weck M, Altintas Y, Beer C (1994) CAD assisted chatter-free NC tool path generation in milling. Int $\mathrm{J}$ Mach Tools Manuf 34 (6):879-891

3. Tobias SA, Fishwick W (1958) A theory of regenerative chatter. The Engineer, London

4. Tlusty J, Polacek M (1963) The stability of machine tools against self excited vibrations in machining. Int Res Prod Eng ASME 465-474

5. Merritt HE (1965) Theory of self-excited machine tool chatter. ASME J Eng Ind 87:447-454

6. Sridhar R, Hohn RE, Long GW (1968) General formulation of the milling process equation. ASME J Eng Ind 90:317-324

7. Slavicek J (1965) The effect of irregular tooth pitch on stability of milling. 6th MTDR Conference Manchester

8. Vanherck P (1967) Increasing Milling Machine Productivity by Use of Cutters with Non-Constant Cutting - Edge Pitch, 8th MTDR Conference Manchester

9. Tlusty J, Koenigsberger F (1970) Machine tool structures, vol. 1, 5th edn. Pergamon, Oxford

10. Opitz H (1968) Chatter Behaviour of Heavy Machine Tools, Quarterly Technical Report No 2 AF 61 (052)-916. Research and Technology Division, Wright Patterson Air Force Base, Dayton

11. Opitz H, Bernardi F (1970) Investigation and Calculation of the Chatter Behaviour of Lathes and Milling Machines. CIRP Ann 18:335-343

12. Minis I, Yanushevsky T (1993) A new theoretical approach for the prediction of machine tool chatter in milling. ASME J Eng Ind 115:1-8

13. Minis I, Yanushevsky T, Tembo R, Hocken R (1990) Analysis of Linear and Nonlinear Chatter in Milling. CIRP Ann 39:459-462

14. Lee AC, Liu CS (1991) Analysis of chatter vibration in the end milling process. Int J Mach Tool Des Res 31(4):471-479

15. Lee AC, Liu CS, Chiang ST (1991) Analysis of chatter vibration in a cutter - workpiece system. Int J Mach Tool Des Res 31 (2):221-234

16. Altintas Y, Budak E (1995) Analytical Prediction of Stability Lobes in Milling. CIRP Ann 44(1):357-362

17. Merdol SD, Altintas Y (2004) Multi Frequency Solution of Chatter Stability for Low Immersion Milling, Journal of Manufacturing Science and Engineering. Trans ASME 126(3):459-466

18. Bravo U, Altuzarra O, Lopez de Lacalle LN, Sanchez JA, Campa FJ (2005) Stability limits of milling considering the flexibility of the workpiece and the machine. Int $\mathrm{J}$ Mach Tools Manuf 45 (15):1669-1680

19. Solis E, Peres CR, Jimenez JE, Alique JR, Monje JC (2004) A new analytical-experimental method for the identification of stability lobes in high-speed milling. Int J Mach Tools Manuf 44 (15):1591-1597

20. Lacerda HB, Lima VT (2004) Evaluation of Cutting Forces and Prediction of Chatter Vibrations in Milling. J Braz Soc Mech Sci Eng 26(1):74-81
21. Budak E, Altintas Y (1998) Analytical Prediction of Chatter Stability in Milling - Part I: General Formulation. Trans ASME 120:22-30

22. Budak E, Altintas Y (1998) Analytical prediction of chatter stability in milling - Part II: application of the general formulation to common milling systems. Trans ASME 120:31-36

23. Campa FJ, Lopez de Lacalle LN, Lamikiz A, Sanchez JA (2007) Selection of cutting conditions for a stable milling of flexible parts with bull-nose end mills. J Mater Process Technol 191(1-3):279282

24. Adetoro OB, Sim WM, Wen PH (2010) Stability lobes prediction for corner radius end mill using nonlinear cutting force coefficients. Mach Sci Technol, in press

25. Adetoro OB, Sim WM, Wen PH (2010) An improved prediction of stability lobes using nonlinear thin wall dynamics. J Mater Process Technol 210(6-7):969-979

26. Thevenot V, Arnaud L, Dessein G, Cazenave-Larroche G (2006) Influence of material removal on the dynamics behavior of thinwalled structures in peripheral milling. Mach Sci Technol 10:275287

27. Seguy S, Campa FJ et al (2008) Toolpath Dependent Stability Lobes for the Milling of Thin-Walled Parts. Int $J$ Mach Machinabil Mater 4(4):377-392

28. Adetoro OB, Wen PH, Sim WM, Vepa R (2009) Numerical and experimental investigation for stability lobes prediction in thin wall machining. Engineering Letters 17(4):257-265, (available at: http:// www.engineeringletters.com/issues_v17/issue_4/EL_17_4_07.pdf)

29. Quintana G, Ciurana J, Teixidor D (2008) A new experimental methodology for identification of stability lobes diagram in milling operations. Int J Mach Tools Manuf 48(6):1637-1645

30. Quintana G, Ciurana J, Ferrer I, Rodríguez CA (2009) Sound mapping for stability lobes diagram identification in milling processes. Int J Mach Tools Manuf 49(3-4):203-211

31. Rayleigh L (1878) Theory of sound, 1945th edition. Dover, New York

32. Caughey TK, O'Kelly MEJ (1960) Classical normal modes in damped linear systems. J Appl Mech 27:269-271

33. Ewins DJ (1984) Modal testing: theory and practice. Research Studies, Letchworth

34. Allemang RJ, Brown DL (1986) Multiple input experimental modal analysis - a survey. Int J Anal Exp Modal Anal 1(1):37-44

35. Mitchell LD (1986) Signal Processing and fast - Fourier transform (FFT) analyzer - a survey. Int J Anal Exp Modal Anal 1 (1):24-36

36. Brown DL (1982) Modal analysis - past, present and future. Proceedings of the International Modal Analysis Conference \& Exhibit

37. Sanliturk KY, Cakar O (2005) Noise elimination from measured frequency response functions. Mech Syst Signal Process 19 (3):615-631

38. Cakar O, Sanliturk KY (2005) Elimination of transducer mass loading effects from frequency response functions. Mech Syst Signal Process 19(1):87-104

39. He J, Fu Z (2001) Modal Analysis. Butterworth-Heinemann, Oxford

40. Altintas Y (2000) Manufacturing automation. Cambridge University Press, New York

41. Grimes RG, Lewis JG, Simon HD (1994) A shifted block lanczos algorithm for solving sparse symmetric generalised eigenproblems. SIAM J Matrix Anal Appl 15:228-272

42. Karlsson \& Sorensen, Inc. (2006) Hibbitt, Abaqus Theory Manual. Karlsson \& Sorensen, Pawtucket 